\title{
Electron Beam Induced Mass Loss Dependence on Stained Thin Epon Resin Sections
}

\author{
Radim Skoupy $^{1}$, Jana Nebesarova ${ }^{2}$ and Vladislav Krzyzanek ${ }^{1}$ \\ 1. Institute of Scientific Instrument, The Czech Academy of Sciences, Brno, Czech Republic. \\ 2. Biology center, The Czech Academy of Sciences, Ceske Budejovice, Czech Republic.
}

Low voltage TEM and STEM (transmission and scanning transmission electron microscope) can be regarded as the method of choice for many structural studies of very thin biological samples like ultrathin sections, viruses etc. [1,2]. Usually, in a conventional TEM (typical acceleration voltage $60-$ $300 \mathrm{kV}$ ) the image contrast is enhanced by staining using salts of heavy metals (e.g., uranyl acetate, lead citrate) [3]. Low voltage STEM allows to image unstained samples that usually show sufficient contrast, but in some cases the staining can be useful. An important parameter for imaging is a sensitivity of the sample to degradation by electron beam. There have been not many studies describing quantitatively the mass loss of the resin sections; the most comprehensive work was performed on the dedicated STEM for molecular mass measurements [4] done at $80 \mathrm{keV}$, or even at higher electron energies done by EFTEM [5]. The author in [6] describes that polymers stained with a heavy metal should therefore damage more rapidly through the secondary electron mechanism and higher cross sections for scattering.

However, there is a lack in such measurements at the energies applied in SEM. The mass loss indicates a degree of the radiation damage; therefore, we report here on investigation of the mass loss of embedding medium (Epon resin of middle hardness) in combination of different thickness of the sections with staining. The main technique used for quantitative measurement of the mass loss is based on the mass measurements by dedicated STEM [7, 8], using SEM equipped by a commercial solid-state STEM detector $[9,10]$. The imaging was performed by SEM Magellan 400L (FEI) at the acceleration voltage of $30 \mathrm{kV}$ and the lowest possible probe current of $1.6 \mathrm{pA}$ in the bright field (BF) mode using the commercial STEM3 detector (FEI). Electron dose for each scan corresponded to about $60 \mathrm{e}^{-} / \mathrm{nm}^{2}$. For our study we used resin sections with thickness 30 and $60 \mathrm{~nm}$. Measurement was performed the second day after their preparation by the ultramicrotome Leica Ultracut UCT. Some slices were stained by uranyl acetate for 30 minutes from the upper side.

Mass loss measurements of the resin slices were performed by imaging the same area at magnification of 100000 times (field of view $1.024 \times 0.884 \mu \mathrm{m}$ ) in a series of many consecutively scanned images (usually 50 first images were recorded). For each experiment, three different places on each sample were imaged for downgrading space dependency of the measurement. Calibration was made using the couple of images recorded for each imaging session, where first of them is absolute dark with beam eclipsed by the grid holder and second one is absolute bright with full beam to the STEM detector. The mass loss was obtained from the normalized BF signals using the Monte Carlo simulation of electron scattering MONCA [11] using the methodology of mass measurements by STEM [12]. All processing was programmed in MATLAB (Mathworks).

As seen on fig. 1 left, the normalized BF signal (showing the fraction of scattered electron to the BF detector) has similar course with nearly linear shape in both thicknesses of the slices without staining; the curves are shifted only by the different thickness. Mass loss (fig. 1 right) shows two groups of thickness independent curves. First one is slice without staining, where at the total irradiation dose of $3000 \mathrm{e} / \mathrm{nm}^{2}$ still remains $64 \%$ of initial mass in the case of $30 \mathrm{~nm}$ and $51 \%$ for $60 \mathrm{~nm}$ slice, respectively. 
However, the slices with staining are clearly much higher sensitive to the electron beam (red and blue circles), where curves in BF signal dependency have an exponential shape. Corresponding mass loss shows the same exponential behavior in both thicknesses. They remain only $21 \%$ of initial mass for both $30 \mathrm{~nm}$ and $60 \mathrm{~nm}$ slices at the same dose of $3000 \mathrm{e} / \mathrm{nm}^{2}$.

We observed higher sensitivity of stained Epon thin sections under incident electron beam. We found change of $43 \%$ mass for $30 \mathrm{~nm}$ and $30 \%$ for $60 \mathrm{~nm}$ slice. This limits the usable dose for imaging by the low voltage STEM (for $30 \mathrm{kV}$ ) because stained sections are more susceptible to burn-out than pure ones.

\section{References:}

[1] H Schatten, JW Jan, A Litwin, Scanning Electron Microscopy for the Life Sciences (2013).

[2] AE Yakushevska et al, Journal of Structural Biology 159 (2007), p. 381-391.

[3] DG Robinson in "Methods of Preparation for Electron Microscopy" (Springer-Verlag, Berlin, 1987).

[4] R Reichelt et al, Ultramicroscopy 16 (1985), p. 69-80.

[5] MA Aronova et al, Journal of Microscopy 239 (2010), p. 223-232.

[6] RF Egerton, Microscopy Research and Techniques 75 (2012), p. 1550-1556.

[7] SA Muller, A Engel, Micron 32 (2001), p. 21-31.

[8] AA Sousa, RD Leapman, Journal of Microscopy 228 (2007), p. 25-33.

[9] M Pfaff et al, Journal of Microscopy 243 (2011), p. 31-39.

[10] T Volkenandt, E. Muller, D. Gerthsen, Microscopy and Microanalysis 20 (2014), p. 111-123.

[11] V Krzyzanek, R. Reichelt, Microscopy and Microanalysis 9 (2003), p. 110-111.

[12] V Krzyzanek et al, Journal of Structural Biology 165 (2009), p. 78-87.

[13] The research was supported by the Grant Agency of the Czech Republic (GA14-20012S), Ministry of Education, Youth and Sports of the Czech Republic (LO1212). The research infrastructure was funded by Ministry of Education, Youth and Sports of the Czech Republic and European Commission (CZ.1.05/2.1.00/01.0017).

a

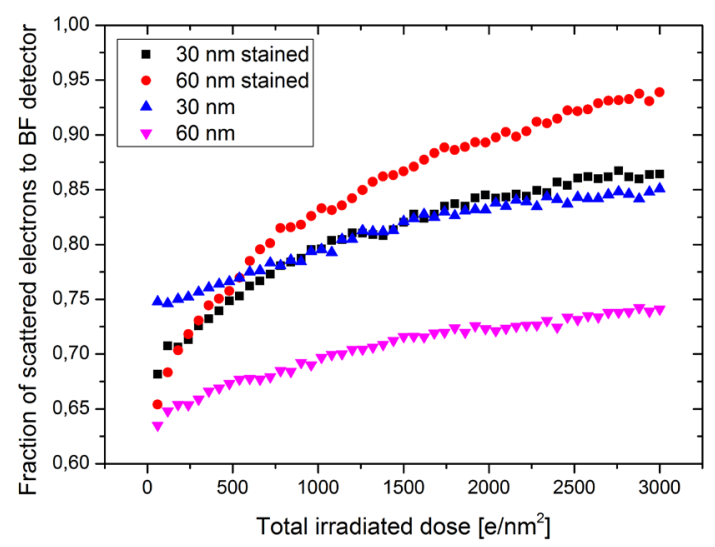

b

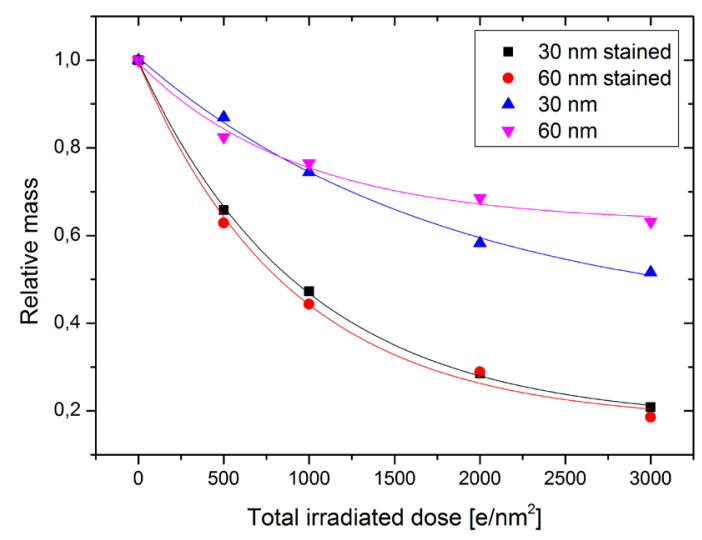

Figure 1. The normalized bright-field (BF) signals (a) and recalculated relative mass (b) dependences on the total irradiation dose on slices of various thicknesses recorded at the second day. 